\title{
Influence of Application of Cold or Heat to the Dog's Body upon the Epinephrine Output Rate.
}

\author{
By \\ SHIDZUKA SAITO.

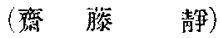 \\ (From the Plysiological Luboratory of Prof. I. Satake, \\ Toholue Imperial University, Sendai.)
}

In cats, the iris of which has been completely denervated by removing the ciliary ganglion besides the superior cervical one, $\mathrm{Hartman}$ with his co-workers ${ }^{1}$ was able to bring about the dilatation of the denervated iris when the animal body was immersed in cold water and the rectal temperature bcgan to fall. If the suprarenals were rendered inactive, cooling was proved to be incapable of causing the paradoxical pupil reaction. The results led them to conclude that stimulation by cold increases pronouncedly the epinephrine discharge ; that this would, in turn, increase heat production if the amount of epinephrine were sufficient, thereby tending to compensate for the loss of heat, was their further suggestion. In the next investigations $\mathrm{F}$. A. Hartman with $W$. B. Hartman ${ }^{2}$ undertook to analyse the mechanism whereby the application of cold upon the animal body augments the epinephrine discharge, and came to witness that it is neither the excitement attendant upon the cooling nor the fall in general body temperature, but the peripheral stimulation of cooling which causes the release of epinephrine. Immersion of cats in water ranging from $39^{\circ}$ to $45^{\circ}$ did not appear to increase the epinephrine discharge. Almost all of the criticisms," made by the Cleveland physiologists on the work of Hartman," are based on the findings in the hands of the latter investigators with the cava pocket method on anaesthetized animals. 64, 1 .

1) F. A. II artman, II. C. McCordock and M. M. Loder, Am. J. Physiol., 1928,

3) F.A. If artman and W. B. Hartman, Ibid., 1923, 65, 612 .

:3) G. N. Stewart and J. M. Rogoff, Ibid., 1928, 66, $257 \mathrm{ff}$. 
The data which corroborate those of Hartman above quoted were published soon afterwards by Cannon. ${ }^{4}$ The denervated heart in the animals surviving and living normally was taken as an indicator of epinephrine discharge. Taking the animal to a cold room, exposing to a cold draught from an open window or introducing cold water into the stomach called forth an aceleration of the denervated heart; when the stuprarenals were rendered inactive these effects did not occur. He inferred that when the animal is exposed to cold, two calorigenic factors, epinephrine and shivering, are at work to protect the body against a fall of body temperature, and that when epinephrine is excluded from action the organism resorts to shivering as it does not when epinephrine is present.

Cannon ${ }^{3)}$ further noted that the denervated heart of cats responded with acceleration when fever was produced by intravenous or intramuscular injection of the suspension of dead typhoid bacilli. After injection cats with intact suprarenals showed first a rapid rise of temperature, the highest being reached in about 34 minutes and amounted to about $1^{\circ} \mathrm{C}$., and then the temperatire usually decreased, but began to rise again slorly and gradually. When the suprarenals were renderel inactive, injection of dead typhoid bacilli brought about only a second slow rise. The increase of denervated heart rate during the first elevation of temperature was too fast for taking the effect of a high body temperature upon the heart itself, on the base of Knowlton and Starling's study of the effect of temperature change on the isolated cat heart.

In this connection the previous findings of $\mathrm{Kun} 0^{\mathrm{B} ;}$ may be recalled here: A bath of $40^{\circ}$ to $45^{\circ}$ accelerates considerably the rate of the denervated heart of rabbits (vagi and accelerantes were divided). An cxcess of 20 per minute in the bath of $40^{\circ}$ and 44 to 45 in that of $45^{\circ}$ was noted.

The conclusion made by Cann on on the effect of fever upon the epinephrine discharge from the suprarenals of cats seemingly contradicts that of $\mathrm{H}$ a $\mathrm{r}$ m an from the experiments on cats in a warm bath from 39 to $45^{\circ}$, provided the acceleration of the denerrated heart and the paradoxical dilatation of the denervated pupil be taken as an unmistakable indication of an increased epinephrine discharge, and further provided a certain portion of the acceleration of the denervated heart in Cannon's experiments be taken as the result of fever itself. In Cat 224 of $\mathrm{Hartm}$ a $\mathrm{n}$ a small increase in the denervated pupil was witnessed when immersed in water of $45^{\circ}$. The rectal temperature increased from $89.0^{\circ}$ to $40.1^{\circ}$. Hartman would explain this increase in the pupil diameter by an increase in the tem-

4) W. B. Cannon and A. Querido, Proc. Nat. Acud. Sci. U. S. A., 1924, 10, 245; W. B. Cannon, A. Querido, S. W. Britton and E. M. Bright, Am. J. Physiol., 19 $(\mathbf{2 6 - )} \mathbf{2 7}, \mathbf{7 9}, 466$.

5) W. B. Cannon and J. R. Pereira, Proc. Nat. Acad. Sci. U. S. A., 1924, 10, 247.

6) Yas Kuno, Pflügers Arch., 1914, 158, 57:. 
perature of the iris. In passing it may be further related that in the previous publications ${ }^{7}$ ) from this laboratory on the epinephrine liberation we found some agents which called forth some rise of the body temperature, but we are at present not able to say definitely the causal relationship between the body temperature rise itself and the augmentation of epinephrine discharge in such cases of toxic fever.

Cram er examined the suprarenal bodies of rats and mice which were exposed to cold, and detected some alterations in the medulla, as depletion of the load of epinephrine, when the body temperature decreased. ${ }^{8}$ ) When mice were exposed to heat epinephrine disappeared sometimes from the periphery of the medulla.. )

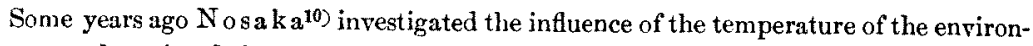
ment upon the epinephrine store of suprarenals of rats. Su to and In ouye's method was applied. The epinephrine content of both the suprarenals was estimated on the mean as $0.00973 \mathrm{grm}$. in toto and $0.45 \mathrm{mgrm}$. per grm. of glands for 15 individuals in the room temperature $\left(19-28^{\circ}\right), 0.00725$ grms. in toto and $0.33 \mathrm{mgrm}$. per grm. for 16 rats which were exposed to cold ( $5^{\circ}$ in the mean) for about two weeks, and $0.01613 \mathrm{mgrm}$. respectively 0.75 mgrm. per grm. for 17 animals which were subjected to a higher temperature such as $35^{\circ}$ for 7 to 9 days and nights. In the assay of the effects of fatigue and temperature on the suprarenals of rats, Vincentii) noted parenthetically that considerable reduction of the temperature of the surrounding medium may, independently of exercise, causes a reduction of the chromaphil reaction, if the animal's temperature becomes lowered; but this does not occur very frequently in the case of white rats.

Since it is alleged that the augmentation of epinephrine discharge and the increase of blood sugar content are closely related with each other, it may not be amiss here to quote the literature concerning the effect of the temperature of the surrounding medium upon the blood sugar content, but at present we may merely point out the papers loaded fully with such kinds of bibliography. A rather complete review of the researches concerning the influence of cooling of animal bodies on the metabolism of carbohydrate is presented in the paper of Morita, ${ }^{12}$ ) which deals with the blood sugar content of cold-punctured rabbits. If a rabbit is punctured between the interbrain and midbrain along the middle line, the body temperature lowers progressively, but until it descends lower than about $30^{\circ}$, the blood sugar level remains unchanged. The previous publications on the effect of a higher surrounding temperature upon the blood sugar are given in the papers of $\mathrm{M}_{\text {o }} \mathrm{rita}^{12}$ ) and $\mathrm{Fuj} \mathrm{ij.}^{13}$ In the heat-punctured rabbits the blood sugar level remains unaffected.14)

'The present writer carried out some investigations along these lines on non-anaesthetized, non-fastened dogs, thereby somewhat different features were recorded; it must however be pointed out that from the previous writers

7) Tadashi Sug a wara, Shidzuka Saito and Mamoru Nemoto, Tohoku J. Exp. Med., 1927, 9, 149; Sugawar a and Hyozo Tada, Ibid., 1927, 9, 295 ; Sugawa ra, Ibid., 1927, 9, 368; Masa. Watanabé, Ibid., 1927, 9, 412; Wa tanabé, Ibid., 1928, 10, 29.

8) W. Cramer, J. Physiol., 19(15-)16, 50, Proc. xxxvirI; Ibid, 1918-19, 52, Proc. VIII ; Sixth Scientific Report of the Imperial Cancer Research Fund, 1919 London, 1.

9) W. Cramer, Brit. J. Exp. Path., 1926, 7, 91.

10) Nosaka (野坂網定), Nihon Naikwagakukwai Zasshi, 1924-25, 12, $473 \mathrm{f}$.

11) S. Vincent, Q. J. Exp. Physiol., 1925, 15, 319.

12) Sachi. Morita, Tohoku J. Exp. Med., 1921, 2, 403.

13) Iju. Fu jii, Ibid., 1922, 3, $88 \mathrm{f}$.

14) Sachi. Morita and Masaru N a ito, Ibid., 1922, 2, 562. 
the animals experimented on were of different kinds in both the series of experiments, the previous and the present, and that the severeness of cooling or heating has assuredly a direct bearing upon the results before us.

\section{Methods.}

Dogs, of which the dorsal roots from IX. thoracic to III. lumbar cord had been previously cut and which were found to have already recovered from the loss of body weight due to the operation, were exclusively used. The lumbar ronte preparation was carried out, and the blood from the suprarenal gland was assayed for the epinephrine content by means of the rabbit intestine segment method. All the procedures and techniques adopted in the present investigations were those given in the previous papers from this laboratory $;^{15}$ only the method of cooling or heating the dog remains to be mentioned. For applying an abnormal temperature to the body surface of the animal a double layered rubber coat was constructed in such a form as to enclose the whole body except the head. For collecting the suprarenal vein blood from both sides and measuring the anal temperature (and for tail) the cont was provided with suitable apertures. In order to let in water one opening near the bottom of every pouch for the limb and three pairs of openings at the dorsal edges of the coat on both sides rrere equipped with rubber tubes, which were usually closed by means of a screwclamp. After putting the animal into the bag coat both the dorsal edges were closed on the dorsal middle line from the neck to the loin by means of cords. In ruming in water the rubber tube or tubes on the limb pouch were comnected with the long limb of the syphon, the other limb of which was dipped in the water bath of a given temperature on a high table, by means of glass and rubber tubes, and the rubber tubes on the other limb pouches were opened, so that air could be got rid of; when the clamps on the rubber tubes connected with the syphon were lifted, water flowed into the double walled bag. In order to allow the water to flow out some rubber tubes on the dorsal edges of the bag and on the limb pouches were opened. The temperature of the water was measured by inserting the thermometer in one of the dorsal openings of the rubber coat.

Before putting the dog into the bag coat, the nails were cut and the paws bandaged in order to protect the rubber bag from scratching. When the bag coat was filled with water the dog became incapable of standing on its feet and lay down; therefore some water was driven out prior to the

15) I. Sataké, T. Sugawara and M. Watanabé, Tohoku J. Fxp. Med., 1927, 8,$501 ;$ 'T. Sugawara, M. Watanabé and S. Saito, Ibid., 1926, 7, $6 \mathrm{ff}$. 
collection of the suprarenal rein blood samples, and they were taken in the proper posture of the animal. When the water in the bag was somewhat hotter, the dog struggled rather violently, so that it was necessary to hold it by the feet and back in order to prevent the bag from tearing, and when very hot water was let into the bag, the mouth of the $\operatorname{dog}$ was tightly bound, else the animal would have bitten the experimentors or the bag.

The use of this rubber bay enabled us to stimulate the animal with at temperature as high as one wishes, but it failed when wished to apply very severe cold; so in some cases a large hole, which corresponded to the form of the trunk of the animal, was made in a large block of ice, and cold was applied by putting the trunk of $\log$ in this hole.

The experimental results are summarized in Tables I and II, the first table is loaded with the results of the application of cold and the second with those of heat. Of one example from each series of experiments the experimental course is given in full account with reproduction of the tracings, but the data of the estimations are omitted here, since they are included in the tables.

Results :

Cold stimulation was applied to five dogs. After completing the lumbar route preparation some samples of the suprarenal vein blood were collected, then the rubber coat was put on, and collection of some samples of the blood from the suprarenal capsule was made. 'The next procedure was the pouring of cold water into the coat. The temperature of the water in the bath was noted, and thereafter that in the coat was also measured from time to time. Some time, as ten to twenty minutes or more, elapsed until the next collection of the blood specimens was conducted, then the coat was taken off. Some minutes later the collection was again made. Such procedures were repeated, or they were followed by cooling of the dog by means of direct contact with the ice block in such a way as is described above. In Dog 5 they were conducted in the reverse order. In one dog, at the termination of the cold experiment, hot water $\left(64^{\circ}\right)$ was let into the coat, this resulted in an acceleration of the rate of epinephrine liberation. It is scarcely necessary to add here that the anal temperature, respiration and hoart beat were measured from time to time.

The temperature of cold water in the bath was adjusted as $3^{\circ}$ to $99^{\circ}$ The anal temperature decreased, but not greatly; in Dog 1 it descended from $40.4^{\circ}$ to $39.2^{\circ}$, in $\operatorname{Dog} 2$ from $39.0^{\circ}$ to $38.5^{\circ}$, in Dog 3 from $38.9^{\circ}$ to $37.8^{\circ}$ (the $\log$ in the coat was placed on an ice block), in Dog 4 from 39.2 to $37.8^{\circ}$ and from $38.6^{\circ}$ to $37.0^{\circ}$ (when laid on ice) and in Dog 5 
from $39.1^{\circ}$ to $37.4^{\circ}$. On cooling the pulse rate invariably increased, while the rhythm of breathing underwent no definite variation, namely in most cases it became slower, but a somewhat faster rate was recorded during the cooling. The dogs shivered, and sometimes cried and struggled.

Now turning to the rate of epinephrine secretion, it may firstly be pointed out, that the epinephrine concentration in the suprarenal vein blood remained completely unaltered in the face of cold stimulation, which affected the body temperature though not so excessively, and secondly that the blood flow through the gland also remained unaffected or increased only a little. As a consequence the velocity with which epinephrine was liberated was quite or nearly equal during the rest spells and the cooling periods. Namely the cooling which was capable of affecting the rectal temperature to decrease to such an extent as $1.2^{\circ}, 0.5^{\circ}, 1.1^{\circ}, 1.5^{\circ}$ and $1.7^{\circ}$ respectively, did not act to influence the rate of epinephrine discharge, contrary to our expectation by analogy of the experiments with cats, carried out by the previous investigators. It may possibly be justifiable to assume that this difference between the present investigations and the previous ones is explained by the difference of the kind of animal. Cats are in general highly sensitive to cold, whereas dogs are not. Working with dogs A a k $\mathbf{i}^{16)}$ and Lépine $e^{1 i}$ were able by cooling to cause glycosuria, appearance of lactic acid in the urine or hyperglycaemia. The body temperature of the dogs of the former investigator decreased remarkably, as to $28-23^{\circ}$ and $26,^{\circ}$ while those of the latter writer had not so low a rectal temperature.

In conclusion of the cooling experiments, it must be added that in Dog 4 the application of hot water $\left(64^{\circ}\right)$ brought about a definite acceleration in the rate of epinephrine output, while the cooling in this dog was proved quite inactive, as in the other dogs.

The heating experiments, the data of which given concisely in Table II, will now be considered. They were carried out on seven dogs, six in Table II and $\operatorname{Dog} 4$ in Table I.

When moderately hot water, as $55^{\circ}$ to $60^{\circ}$, was poured into the coat, the animal looked quite comfortable though the breathing becane faster and the conjunctiva hyperaemic. The pulse rate decreased invariably. The temperature of water in the cont was estimated as $43^{\circ}, 45^{\circ}$ and so forth. The anal temperature increased a little (in $\operatorname{Dog} 4$ from $38.4^{\circ}$ to $40.4^{\circ}$, in $\operatorname{Dog} 6$ from $39.2^{\circ}$ to 40.5 , in $\operatorname{Dog} 7$ from $38.4^{\circ}$ to $40.6^{\circ}$, in $\operatorname{Dog} 8$ from $38.5^{\circ}$ to $40.5^{\circ}$ and in Dog 11 from $38.7^{\circ}$ to $40.8^{\circ}$ ).

16) T. Araki, Hoppe-Sey ler's Ztschr., 1892, 16, $457 \mathrm{f}$.

17) R. Lépine, Le diabète sucré, Paris 1909, 192. 


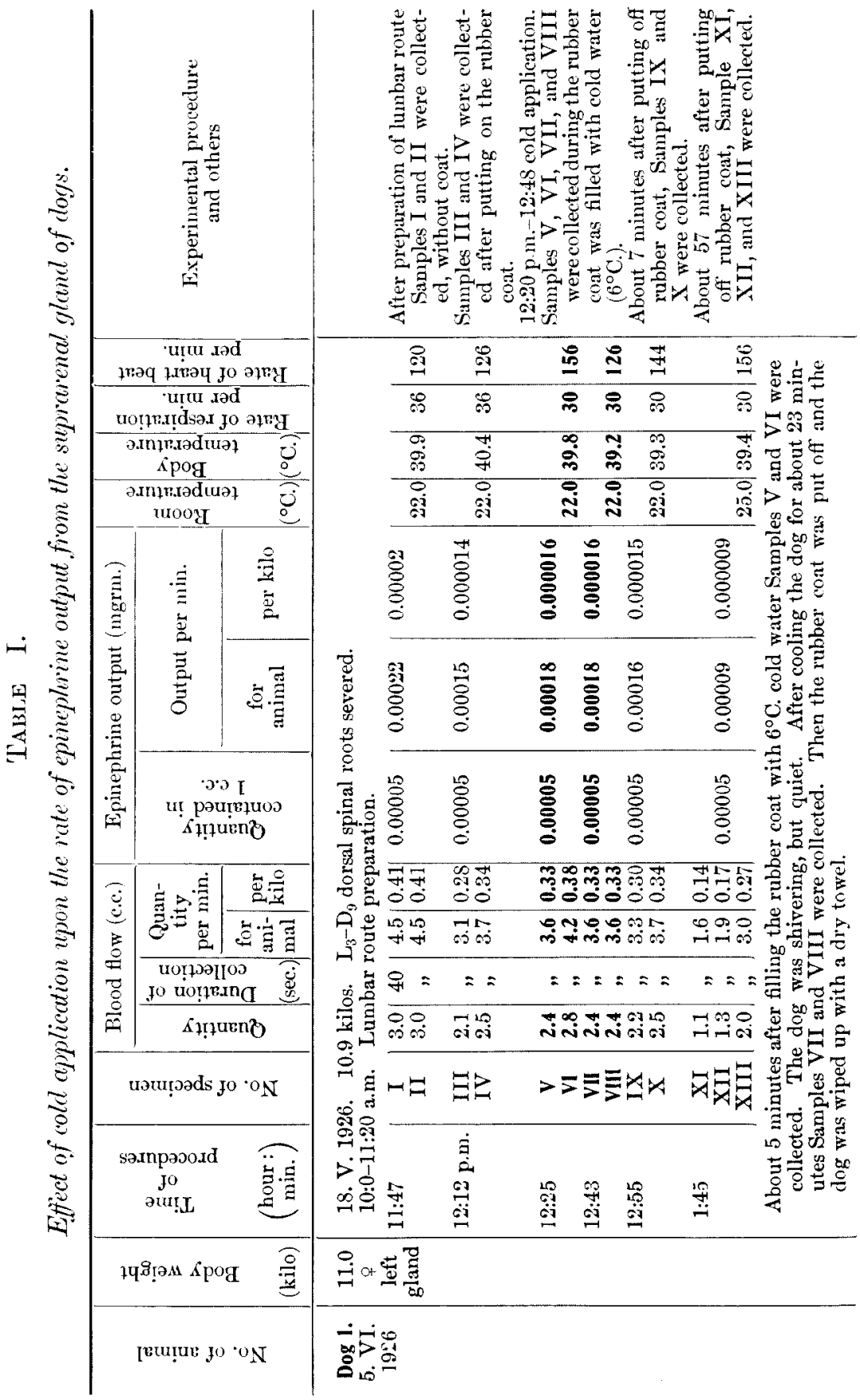




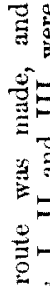
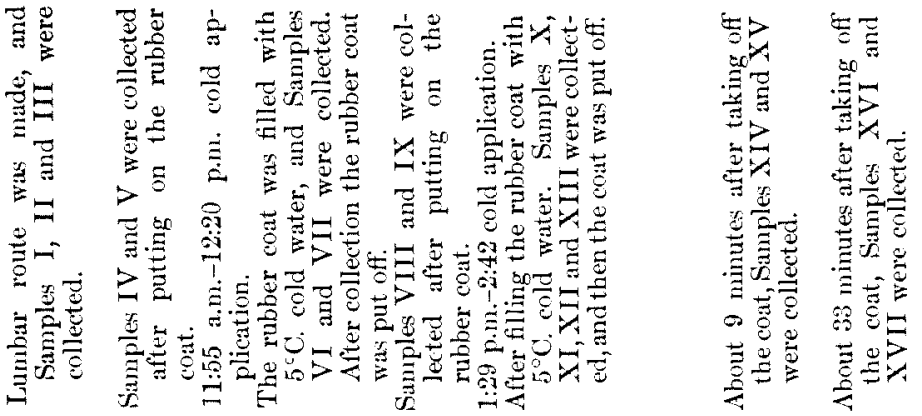

\begin{tabular}{|c|c|c|c|c|c|c|c|}
\hline 8 & 量 & 보 & 8 & $\stackrel{\infty}{2}$ & $\frac{\alpha}{a}$ & $\stackrel{g}{g}$ & 塪二. \\
\hline I & $\overrightarrow{0}$ & $\ddot{\sim}$ & $\stackrel{\infty}{\pi}$ & $\vec{N}$ & $\alpha$ & $\Phi$ & $\overrightarrow{0}$ \\
\hline & 居 & : & $\stackrel{8}{8}$ & 邑 & $\ddot{g}$ & $\frac{20}{00}$ & $\overrightarrow{\partial j}$ \\
\hline 8 & $\stackrel{0}{\dot{j}}$ & வ. & $\stackrel{\infty}{g}$ & : & 5 & $\stackrel{\circ}{\circ}$ & \\
\hline
\end{tabular}

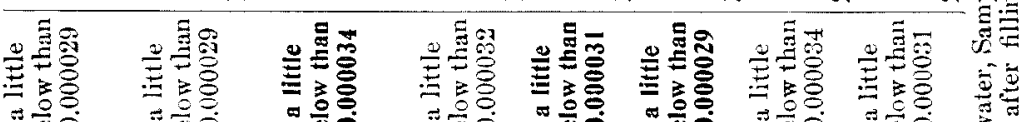

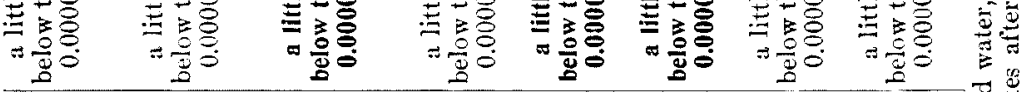

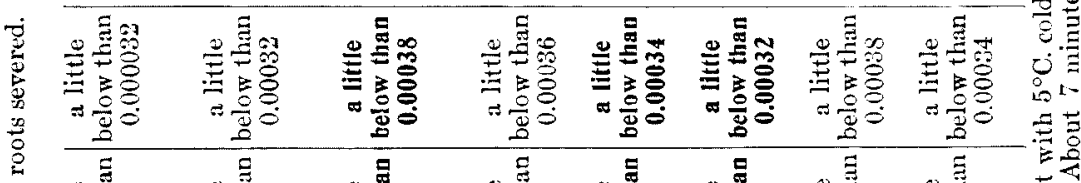

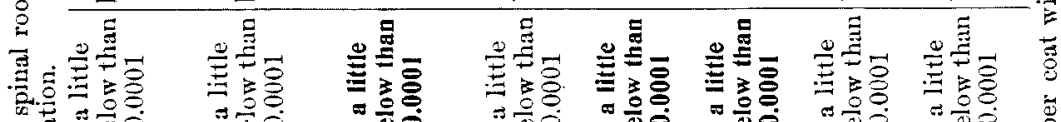

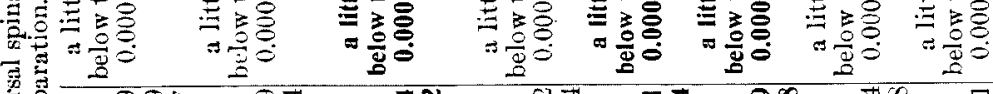

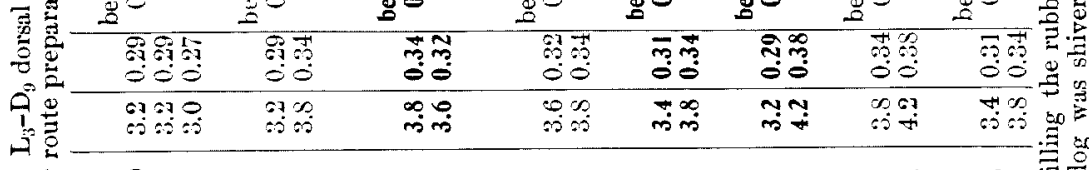

\begin{tabular}{|c|c|c|c|c|c|c|c|}
\hline $\bar{B}==$ & $==$ & $==$ & $==$ & $\therefore=$ & $\therefore=$ & $==$ & $==\frac{\pi}{4}$ \\
\hline 900 & $\stackrel{\circ}{\circ}$ & $9 \infty$ & mo & $=9$ & $\underline{-\bar{x}}$ & $\overrightarrow{S i}$ & 190 \\
\hline$-\Xi \Xi$ & $\exists$ & $\equiv \equiv$ & $\exists A$ & $x \bar{x}$ & $\overline{\bar{x}} \overline{\bar{x}}$ & 总学 & \\
\hline$\stackrel{\text { ạ }}{=}$ & $\stackrel{9}{=}$ & $\begin{array}{l}\dot{\Xi} \\
\vdots \\
亡 \\
\ddot{\Xi}\end{array}$ & $\Xi$ & 兽 & 瓷 & $\overrightarrow{2}$ & $\stackrel{\frac{12}{ت}}{\because: 0}$ \\
\hline
\end{tabular}

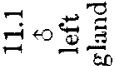




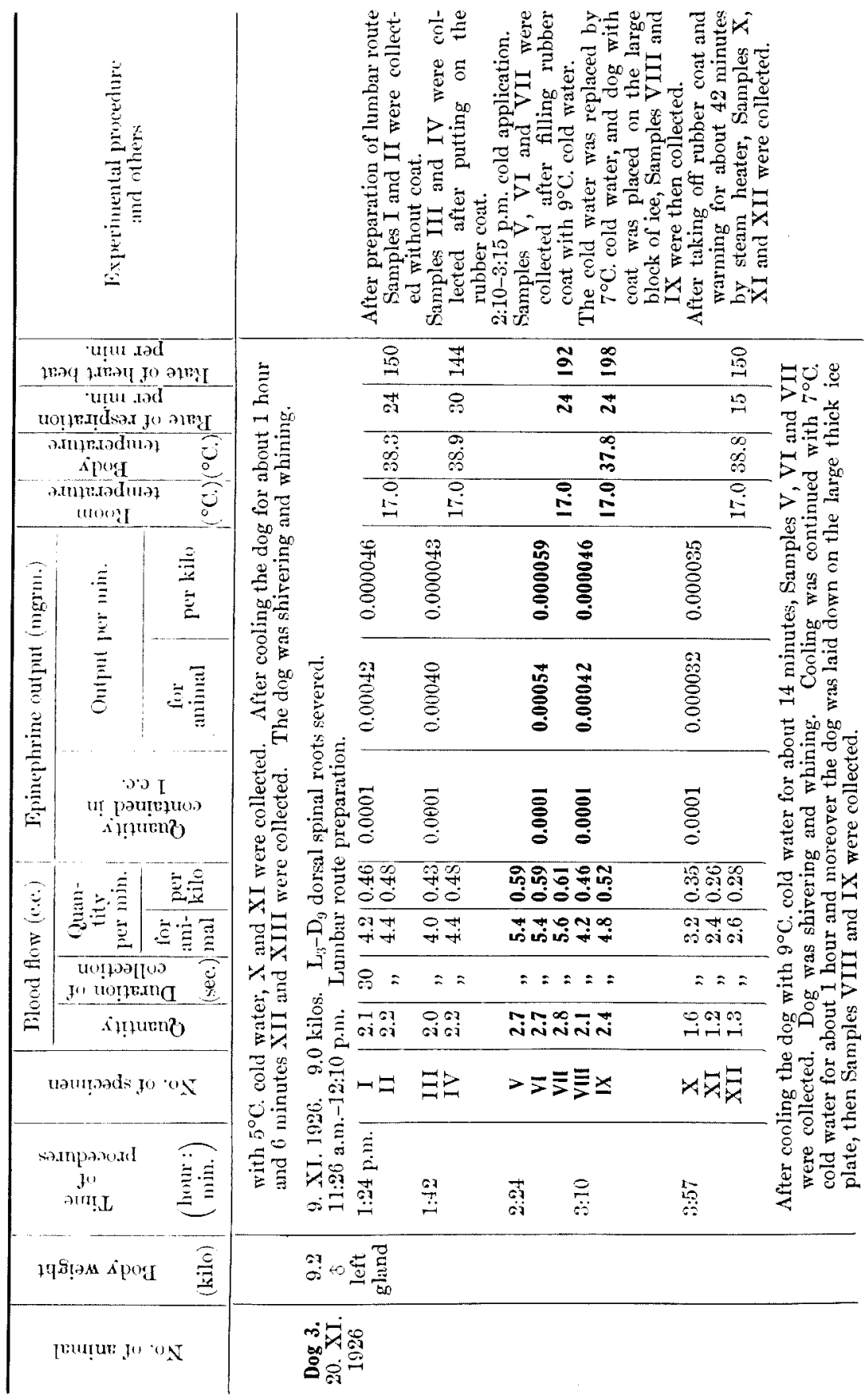




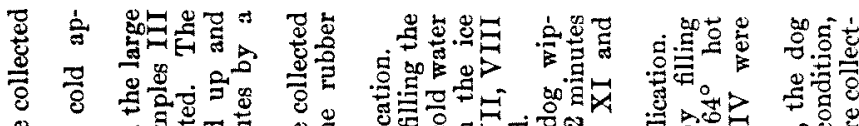

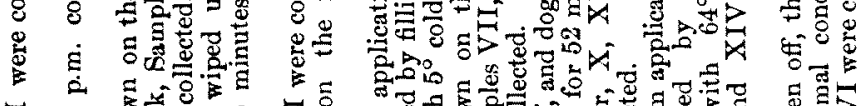
ஏ

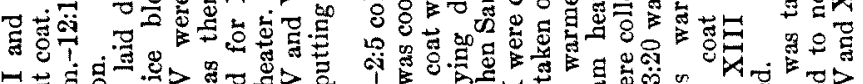

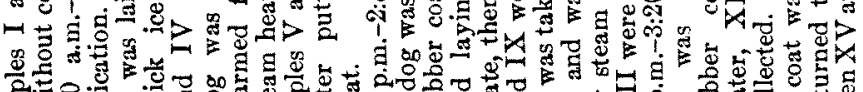

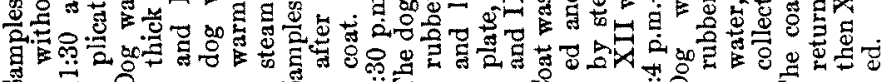
政 员

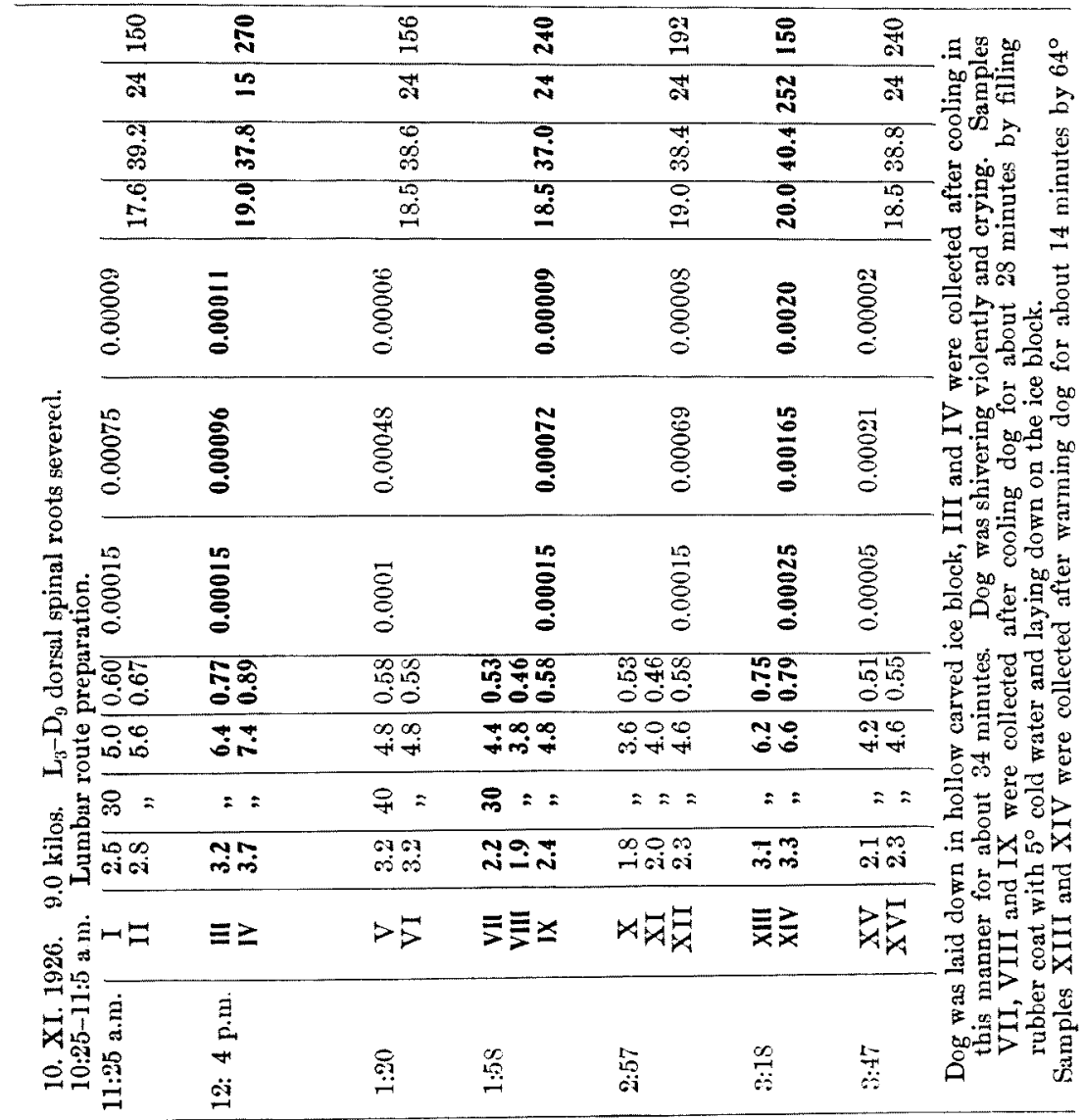

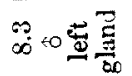




\begin{tabular}{|c|c|c|c|c|c|c|c|c|c|}
\hline & 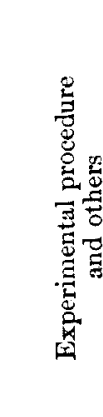 & & & & 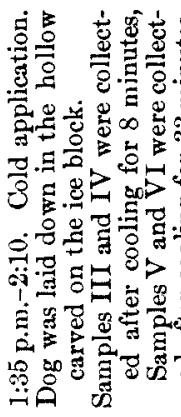 & 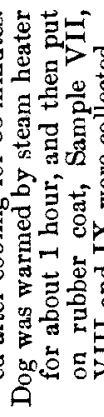 & 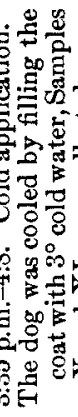 & 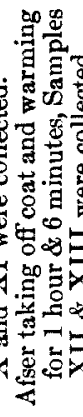 & \\
\hline & 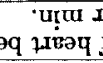 & วluy & & & 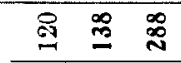 & $\stackrel{8}{\mathscr{9}}$ & $\underset{N}{\mathbb{N}}$ & 昌 & 田ざ \\
\hline & 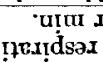 & $\begin{array}{l}\text { d } \\
\text { o әرEY }\end{array}$ & $\stackrel{50}{8}$ & & G゙ & 8 & $\bar{N}$ & कि & \\
\hline & $\begin{array}{l}\text { Intexadu } \\
\text { रрog }\end{array}$ & 10 & 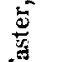 & & $\begin{array}{ll}10 & + \\
00 & 0 \\
80\end{array}$ & $\overline{0}$ & $\frac{\pi}{m}$ & $\begin{array}{l}1 \\
\infty \\
\infty\end{array}$ & 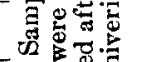 \\
\hline & $\begin{array}{l}\text { Injexada } \\
\text { uooy }\end{array}$ & $7 \quad 3$ & $\stackrel{\Xi}{\Xi}$ & & 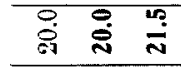 & 虽 & वें & $\stackrel{8}{0}$ & 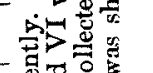 \\
\hline 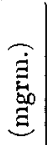 & 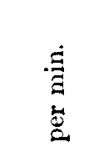 & 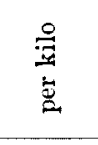 & 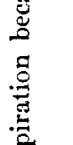 & & 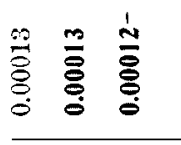 & $\frac{1}{3}$ & $\overline{\overline{8}}$ & $\begin{array}{l}\mathscr{8} \\
\stackrel{8}{8} \\
\dot{0}\end{array}$ & 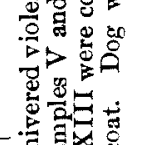 \\
\hline 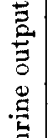 & $\frac{\vec{Z}}{\overrightarrow{3}}$ & 总. & 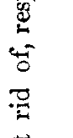 & 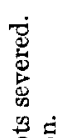 & 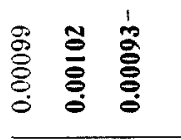 & 范 & $\stackrel{\infty}{0}$ & $\begin{array}{l}0 \\
8 \\
8 \\
8\end{array}$ & 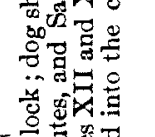 \\
\hline 咅 & 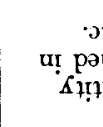 & $\begin{array}{l}\text { L } \\
\text { [8+uos } \\
\text { eno }\end{array}$ & 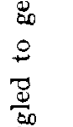 & 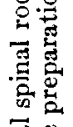 & 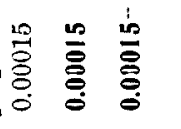 & 占 & 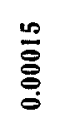 & $\frac{10}{3}$ & 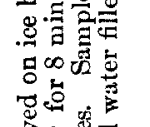 \\
\hline 3 & $\dot{\Xi} \therefore$ & $\stackrel{9}{\Xi}$ & 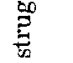 & 莡苋 & 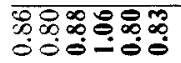 & 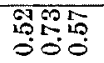 & 몽 & $\begin{array}{l}190 \\
000\end{array}$ & 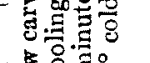 \\
\hline 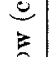 & $\vec{\jmath} \Xi$ & 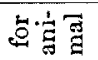 & ] & คํี & 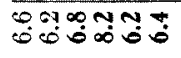 & OP & की & Sid & 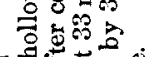 \\
\hline 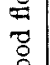 & $\begin{array}{l}\text { uotpo } \\
\text { Jo บัตุ? }\end{array}$ & in & $\begin{array}{l}E \bar{O} \\
\overrightarrow{0}\end{array}$ & 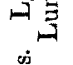 & $8=:=:=$ & $==2$ & $==$ & $F=$ & 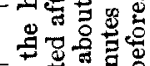 \\
\hline$\vec{n}$ & K†! & $\cos$ & $\begin{array}{ll}00 \\
00\end{array}$ & $\stackrel{0}{3}$ & 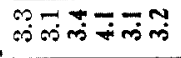 & $\begin{array}{l}0 \text { o o } \\
\text { oi oj }\end{array}$ & nin & Gis & 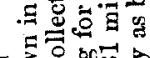 \\
\hline & 」ашụว & $0.0 \mathrm{~N}$ & 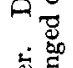 & $\infty$ & 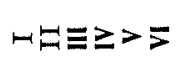 & $\Xi \Xi$ & $x \bar{x}$ & 氙氙 & 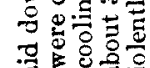 \\
\hline & $\begin{array}{l}\text { Inpoooxd } \\
\text { jo } \\
\text { ərut.d }\end{array}$ & $\overparen{\ddot{\Xi} \cdot \dot{\Xi}}$ & 胥 & 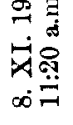 & 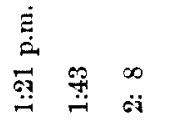 & $\stackrel{10}{\circ}$ & $\begin{array}{l}0 \\
\ddot{\forall}\end{array}$ & $\underset{\ddot{B}}{\stackrel{\#}{*}}$ & 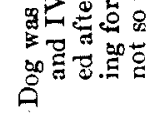 \\
\hline & 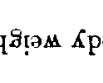 & d $\stackrel{\widehat{0}}{\underline{y}}$ & & & 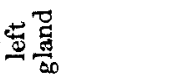 & & & & \\
\hline & [8tutas & & & 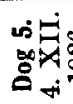 & & & & & \\
\hline
\end{tabular}


Influence of Cold or Heat upon Epinephrine Output of Dogs

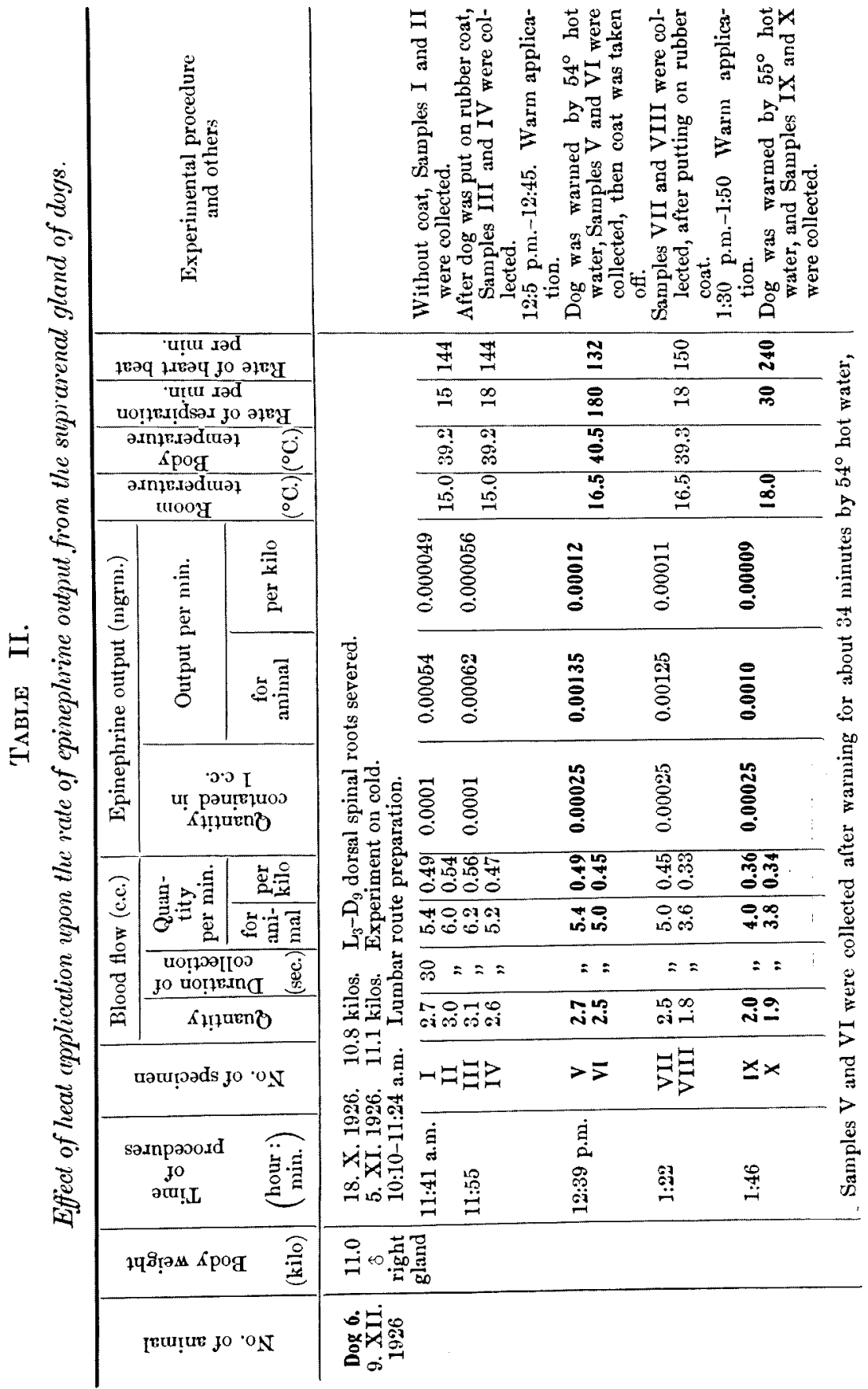




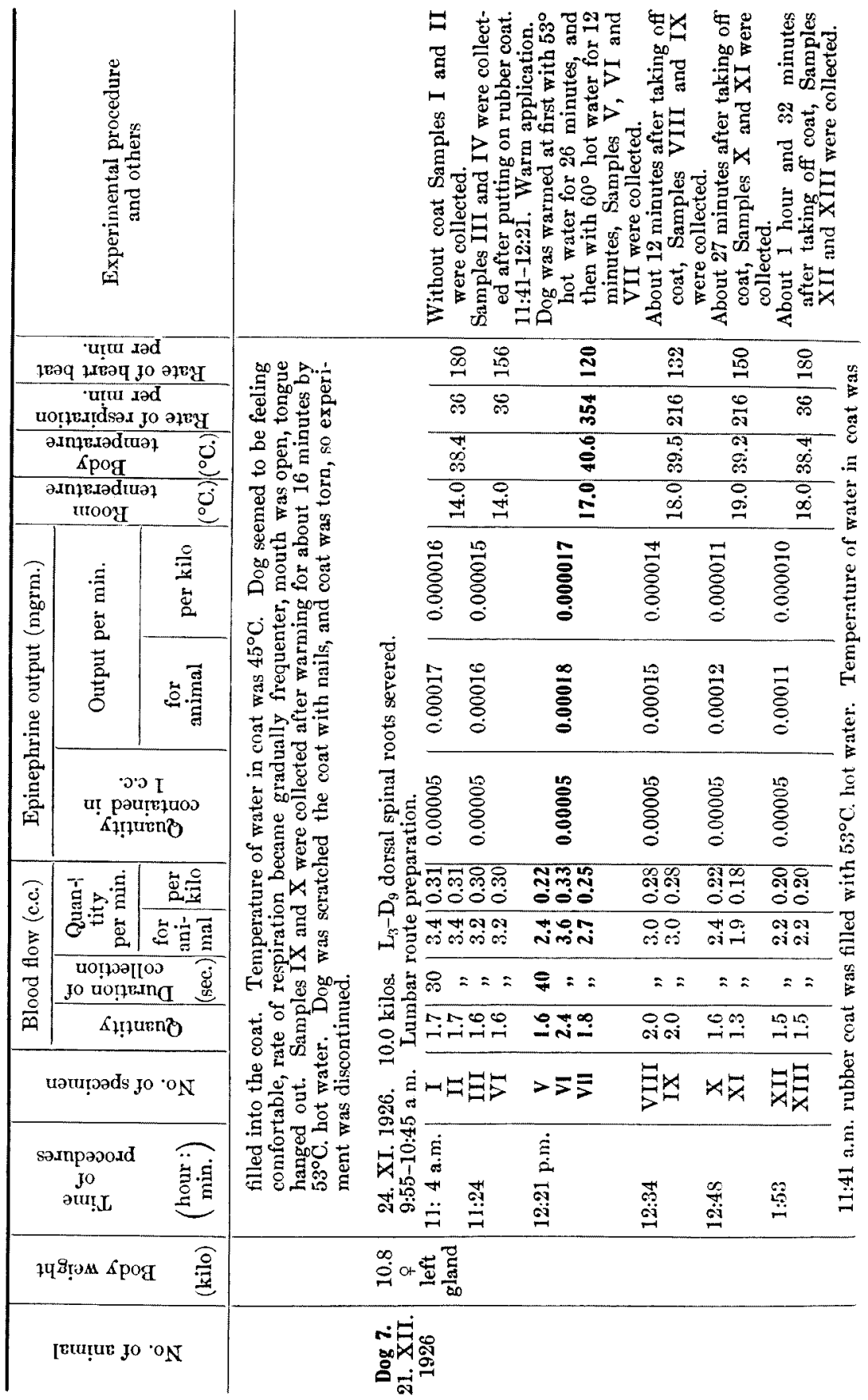



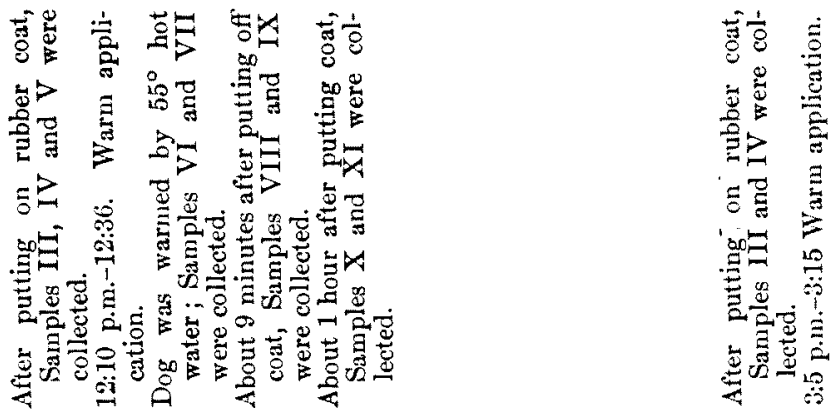

器繁

\begin{tabular}{|c|c|c|c|c|}
\hline $\begin{array}{l}30 \\
0\end{array}$ & 8 & 8 & 畠 & \pm \\
\hline$\infty$ & $\overrightarrow{G i}$ & $\bar{z}$ & $\infty$ & $\overrightarrow{B J}$ \\
\hline
\end{tabular}

总离

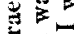

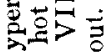

=

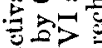

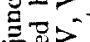

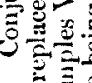

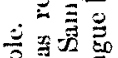

$\exists$ 过

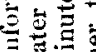

翌百

可些哥

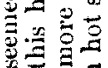

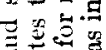

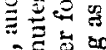

\begin{tabular}{|c|c|c|c|c|}
\hline $\begin{array}{l}10 \\
\infty\end{array}$ & 10 & $\stackrel{\infty}{\theta}$ & $\stackrel{9}{9}$ & $\vec{\infty}$ \\
\hline $\begin{array}{l}10 \\
0 \\
0\end{array}$ & 8 & $\stackrel{\overrightarrow{0}}{0}$ & 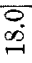 & $D_{0}$ \\
\hline
\end{tabular}

苛魚焉

잉

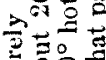

昰定 $x_{1}: 3$

a 00

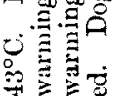

$\sum_{8}$

总

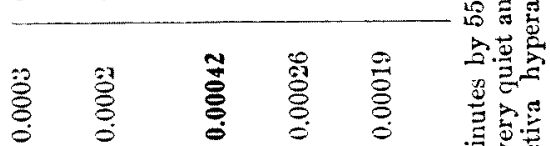

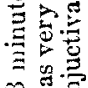

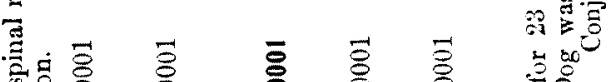

焉事

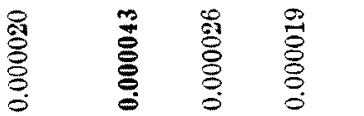

疍

范.

is 3

营

家员要

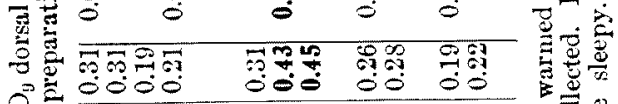

A 0007

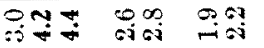

过

总

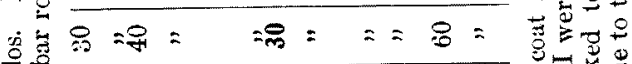

可产

요문

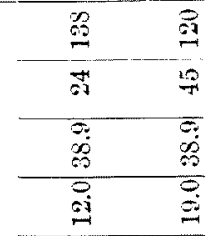

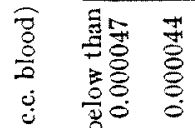

象过

8.9

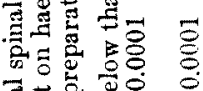

要茫要员

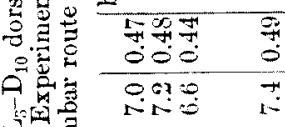

宛实= =

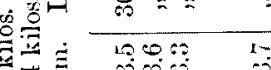

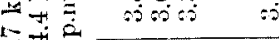

官

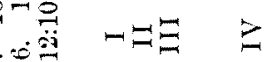

跑

車产

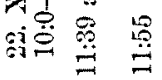

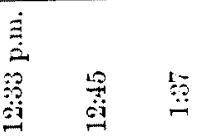

की

家宁

望

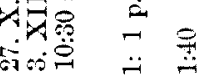

$\stackrel{\circ}{\circ}+\stackrel{g}{\Xi}$

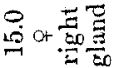

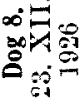

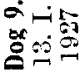




\begin{tabular}{|c|c|c|c|c|c|c|c|c|c|c|c|c|}
\hline & 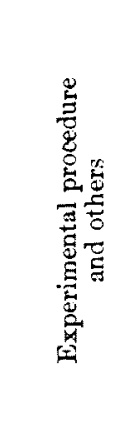 & & 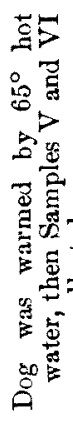 & 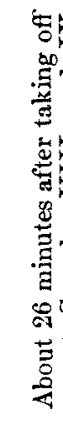 & 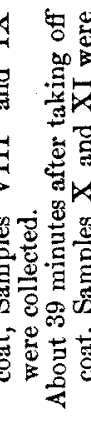 & & & & 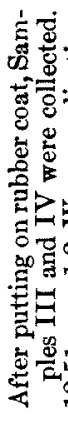 & 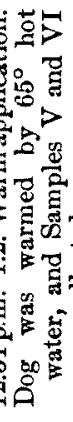 & 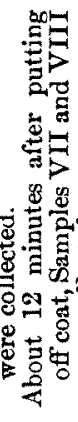 & 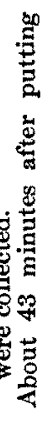 \\
\hline qeo & 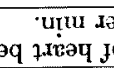 & d & $\stackrel{9}{\simeq}$ & $\underset{\substack{\infty \\
\hdashline}}{0}$ & $\stackrel{9}{\circ}$ & 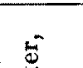 & & 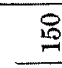 & $\underset{0}{\infty}$ & ㅍ & శ్ & \\
\hline & $\begin{array}{l}\text { 'untor do } \\
\text { yextdsax }\end{array}$ & 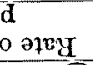 & 록 & 97 & 8 & & & $\infty$ & $\frac{\infty}{7}$ & 疋 & กิ & \\
\hline & $\begin{array}{l}\text { Inpsadua } \\
\kappa \mathrm{pog}\end{array}$ & $7 \longdiv { 0 }$ & $\stackrel{n}{\mathscr{2}}$ & & & $\frac{D^{5}}{4}$ & & 吕 & o & $\stackrel{m}{=}$ & @़̣ & \\
\hline & $\begin{array}{l}\text { minuadu } \\
\text { tuooy }\end{array}$ & 90 & $\stackrel{\infty}{=}$ & $\stackrel{0}{\infty}$ & \begin{tabular}{l}
0 \\
$\infty$ \\
\hdashline
\end{tabular} & 号点 & & 0 & 5 & $\stackrel{\infty}{\infty}$ & $\begin{array}{l}\infty \\
= \\
\end{array}$ & \\
\hline 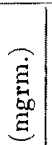 & 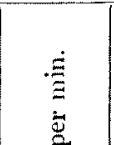 & 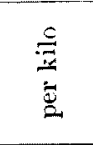 & 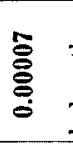 & $\begin{array}{l}\text { in } \\
\stackrel{9}{8} \\
8 \\
0\end{array}$ & $\begin{array}{l}10 \\
8 \\
8 \\
8 \\
8 \\
8 \\
8\end{array}$ & 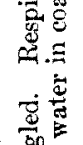 & & $\begin{array}{l}\stackrel{+}{8} \\
\dot{\delta} \\
\\
\end{array}$ & $\begin{array}{l}\stackrel{9}{8} \\
\stackrel{8}{8} \\
8 \\
0\end{array}$ & $\stackrel{\mathscr{O}}{\stackrel{\Xi}{\Xi}}$ & $\frac{1}{8}$ & \\
\hline 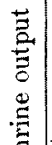 & $\bar{\Xi}$ & E. & $\bar{\Phi}$ & 8 & 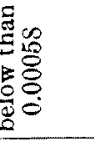 & 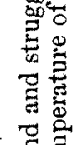 & 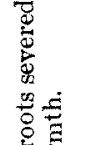 & $\begin{array}{l}10 \\
\stackrel{8}{8} \\
8 \\
8\end{array}$ & $\begin{array}{l}\frac{5}{8} \\
8 \\
8 \\
0\end{array}$ & $\stackrel{\widetilde{\Xi}}{\stackrel{\Xi}{0}}$ & $\frac{\sigma}{8}$ & \\
\hline 总 & $\begin{array}{r}0 \\
\text { uा pau } \\
\dot{x} \geqslant \pm 0\end{array}$ & $\begin{array}{l}\text { T } \\
\text { !epuos } \\
\text { en? }\end{array}$ & 离. & 8 & $\begin{array}{l}5 \\
\overline{8} \\
8 \\
8\end{array}$ & 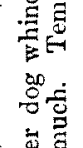 & 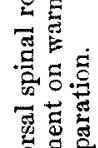 & & 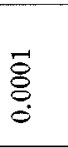 & $\stackrel{\check{E}}{\circ}$ & $\stackrel{8}{\mathscr{E}}$ & \\
\hline சे & $\dot{\Xi} \Rightarrow . \dot{\Xi}$ & 产要 & 突呫 & वृ & $\begin{array}{l}80 \\
80 \\
80\end{array}$ & 葛导 & 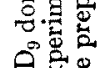 & 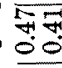 & क्ष & $\underset{\substack{\infty \\
0}}{\infty}$ & है: & $\begin{array}{l}\infty \\
0 \\
0\end{array}$ \\
\hline $\begin{array}{l}\dot{U} \\
\end{array}$ & 焉焉 & Eั ฮี & $\pi y$ & $\begin{array}{ll}\infty & 9 \\
0 & 0 \\
0\end{array}$ & D. & 苍焉 & 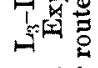 & ธุำ & $\stackrel{\infty}{\infty}+0$ & yy & sio & $\stackrel{9}{4}$ \\
\hline$\frac{\Xi}{8}$ & $\begin{array}{l}\text { uo!porl } \\
\text { jo vo!̣? }\end{array}$ & $\mathrm{n}_{\mathrm{O}} \mathrm{S}$ & ల్ : & 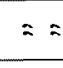 & - & E.气 & 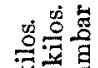 & $8:$ & $=2=$ & $==$ & $==$ & $=$ \\
\hline 9 & $A_{1} ! \mathrm{U}$ & 80 & & $\$ 0$ & $\underset{s i c j}{0}$ & $\stackrel{8}{\mathscr{E}}$ & 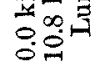 & $\begin{array}{r}000 \\
0100 \\
\end{array}$ & ing & $\bar{N} \bar{N}$ & $\stackrel{\circ}{\rightarrow} \stackrel{0}{\sim}$ & ai \\
\hline & Hauraods J & $O \cdot O N$ & $>5$ & 国 & $\triangle A$ & 蛋 & 형 & & $\Xi 5$ & $>5$ & 且巨 & 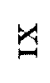 \\
\hline$s 9$. & $\begin{array}{l}\text {.mparoxd } \\
\text { Jo } \\
\text { əư!L }\end{array}$ & $\overparen{\Xi}$ & $\stackrel{10}{60}$ & $\frac{97}{40}$ & $\frac{12}{60}$ & ${ }_{巳}^{8}$ & 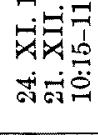 & 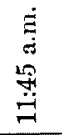 & 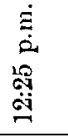 & $\begin{array}{l}61 \\
\ddot{-1}\end{array}$ & $\stackrel{5}{ت 7}$ & 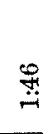 \\
\hline & q & $\mathrm{A} \stackrel{\mathrm{g}}{\mathrm{E}}$ & & & & & 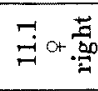 & & & & & \\
\hline & [ruture Jo & $\mathrm{N}$ & & & & & $\begin{array}{l}9 \\
80\end{array}$ & & & & & \\
\hline
\end{tabular}




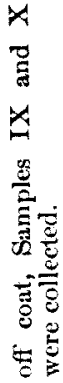

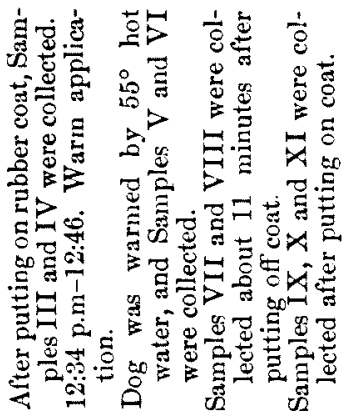

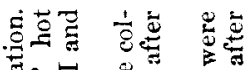

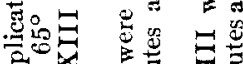

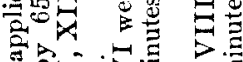

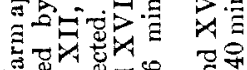

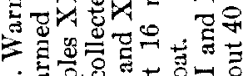

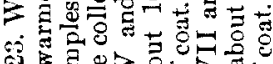

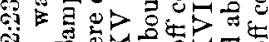

i g o

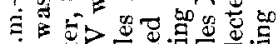

(

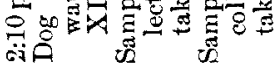

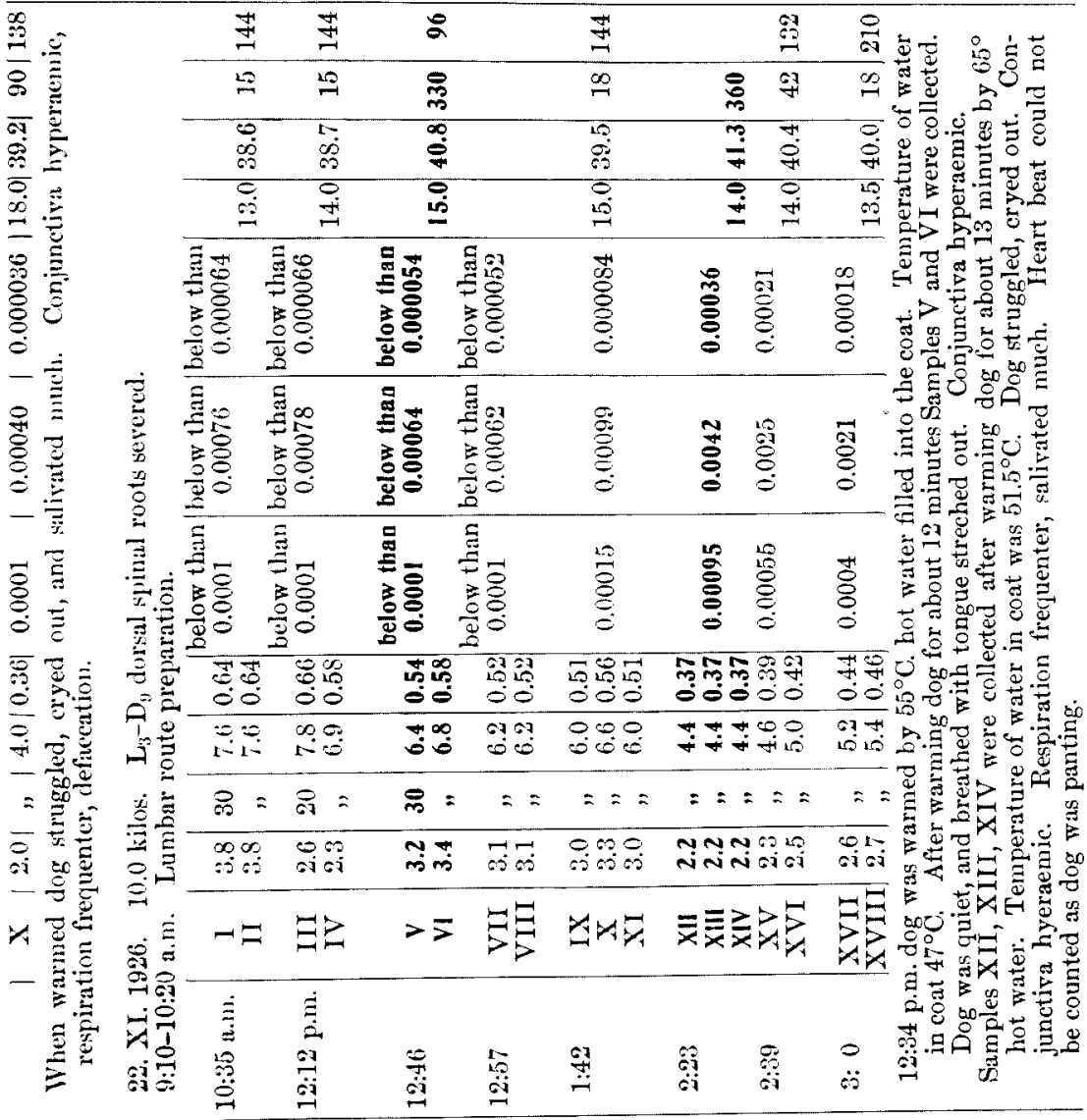

$\stackrel{\infty}{=} c+\underline{\underline{G}}$

=-ir 
Example I.

Dog 5 . 우

8. XI. 8.5 kilos. $\mathrm{L}_{3}-\mathrm{D}_{9}$ dorsal spinal roots severed.

4. XII. 7.7 kilos.

\begin{tabular}{|c|c|c|c|c|c|}
\hline Time & $\begin{array}{l}\text { Room } \\
\text { temper- } \\
\text { ature } \\
\left({ }^{\circ} \mathrm{C}\right)\end{array}$ & $\begin{array}{l}\text { Body } \\
\text { temper- } \\
\text { ature } \\
\left({ }^{\circ} \mathrm{C}\right)\end{array}$ & $\begin{array}{c}\text { Fre- } \\
\text { quency } \\
\text { of } \\
\text { respira- } \\
\text { tion } \\
\left(\begin{array}{c}\text { per } \\
\text { min. }\end{array}\right)\end{array}$ & $\begin{array}{c}\text { Rate of } \\
\text { heart } \\
\text { beat }\end{array}$ & Experimental process and others \\
\hline $\begin{array}{l}10: 40 \mathrm{a} \cdot \mathrm{m} \\
11: 30 \\
12: 0 \mathrm{~m}\end{array}$ & 18.0 & 38.8 & 21 & 96 & $\begin{array}{l}\text { Dog was taken to operation room. } \\
\text { Lumbar route operation started. } \\
\text { Central end of l. suprarenal vein was } \\
\text { prepared, and a somewhat thick } \\
\text { and long thread passed under it. }\end{array}$ \\
\hline 12:20 p.m. & 19.0 & 38.8 & 24 & 126 & $\begin{array}{l}\text { Small cannula was inserted into peri- } \\
\text { pheral end of l. suprarenal vein. }\end{array}$ \\
\hline $\begin{array}{l}1: 21 \\
1: 35\end{array}$ & 20.0 & 38.5 & 24 & 120 & $\begin{array}{l}\text { Specimens I and II were collected. } \\
\text { Laid dog in a hole carved on a block } \\
\text { of ice. }\end{array}$ \\
\hline $1: 41$ & & & & & Dog shivered markedly. \\
\hline $1: 43$ & 20.0 & & 24 & 138 & $\begin{array}{l}\text { Specimens III and IV were collect- } \\
\text { ed. }\end{array}$ \\
\hline $1: 50$ & & & & & $\begin{array}{l}\text { Dog was shivering more violently, } \\
\text { whining. }\end{array}$ \\
\hline $\begin{array}{l}1: 53 \\
2: 5\end{array}$ & $\begin{array}{l}21.0 \\
21.0\end{array}$ & $\begin{array}{l}38.2 \\
38.2\end{array}$ & 15 & 198 & \\
\hline $\begin{array}{l}2: 8 \\
2: 10\end{array}$ & 21.5 & 38.4 & 12 & 288 & $\begin{array}{l}\text { Specimens V and VI were collected. } \\
\text { Dog was wiped up with a dry towel, } \\
\text { and brought near steam heater. }\end{array}$ \\
\hline $\begin{array}{l}3: 10 \\
3: 17\end{array}$ & 22.0 & 39.1 & 30 & 120 & $\begin{array}{l}\text { Rubber coat was put on dog. } \\
\text { Specimens VII, VIII and IX were } \\
\text { collected. }\end{array}$ \\
\hline $8: 30$ & & & & & $\begin{array}{l}3^{\circ} \mathrm{C} \text { cold water was flowed into coat } \\
\text { and it was filled in } 5 \text { minutes. } \\
\text { Dog was shivering, but not so violent- } \\
\text { ly as before. She was whining and } \\
\text { grawing operation table. }\end{array}$ \\
\hline 4: 0 & 20.0 & 37.4 & 24 & & \\
\hline $\begin{array}{l}4: 6 \\
4: 8\end{array}$ & 20.0 & 37.4 & 24 & 270 & $\begin{array}{l}\text { Soon after drawing off coat Speci- } \\
\text { mens X and XI were collected. } \\
\text { Dog was wiped up carefully with dry } \\
\text { towels. }\end{array}$ \\
\hline $5: 14$ & 16.0 & 38.7 & 30 & 150 & $\begin{array}{l}\text { Specimens XII and XIII were col- } \\
\text { lected. }\end{array}$ \\
\hline $\begin{array}{l}5: 45 \\
6: 0\end{array}$ & & & & & $\begin{array}{l}\text { R. femoral artery was prepared, and } \\
\text { about } 100 \text { c.c. blood was bled as a } \\
\text { indifferent blood in epinephrine } \\
\text { estimation. } \\
\text { Wounds sewed up. } \\
\text { Dog was brought back to kennel. }\end{array}$ \\
\hline
\end{tabular}




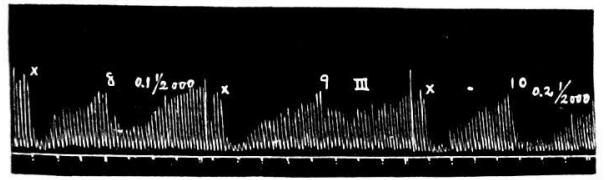

Fig. 1. a. III : Decidedly stronger than $0.00005 \mathrm{mgrm}$; far weaker than $0.0001 \mathrm{mgrm}$.

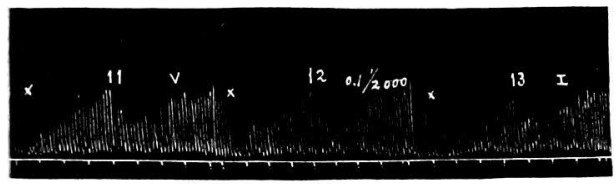

Fig. 1. b. V: Far weaker than 0.0001 mgrm. (Obs. 10); a little stronger than 0.00005 mgrm. I : Stronger than $0.00005 \mathrm{mgrm}$.

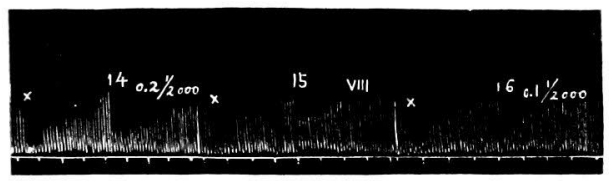

Fig. 1. c. VIII : Weaker than $0.0001 \mathrm{mgrm}$; ; almost as strong as or a little stronger than $0.00005 \mathrm{mgrm}$. I : Very far weaker than $0.0001 \mathrm{mgrm}$. (Obs. 13).

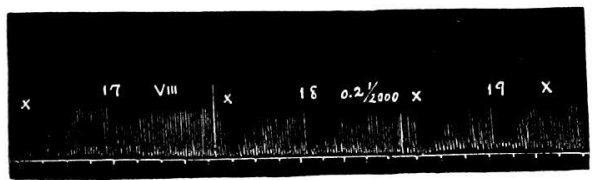

Fig. 1. d. VIII : Stronger than $0.00005 \mathrm{mgrm}$. (Obs. 16); weaker than $0.0001 \mathrm{mgrm}$. $\mathrm{X}$ : Weaker than 0.0001 mgrm.

* In all the intestine tracings, at the mark " " atropine-T y rode's solution, in which the rabbit intestine segment was beating rhythmically, was replaced by indifferent blood solution, and at the "numeral" the indifferent blood solution was displaced by adrenaline blood solution, that is, the indifferent blood solution to which a certain quantity of adrenalin chloride of $\mathrm{Sa} \mathbf{n} \mathrm{k}$ y o Co. was added, or by the suprarenal vein blood specimen solution. All the blood solutions were prepared by diluting with 4 volumes of Ty rod e's solution and the quantity of the bloods employed for one assay was 0.5 c.c. The numeral of suprarenal vein blood specimens and the quantity of adrenaline hydrochloride solution which was indicated by c.c. and concentration were noted on each tracing. For example "III" represents the III. suprarenal vein blood specimen, and " $0.11 / 2000$ " shows " 0.1 c.c. of adrenaline hydrochloride solution with a concentration of $1 / 2000 \mathrm{mgrm}$. in 1 c.c." i. e. 0.00005 mgrm.

In all tracings, time intervals 30 seconds. 


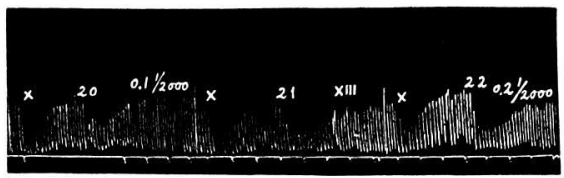

Fig. 1. e. X : Clearly stronger than $0.00005 \mathrm{mgrm}$. (Obs. 19). XIII : Far stronger than $0.00005 \mathrm{mgrm}$; weaker than $0.0001 \mathrm{mgrm}$.

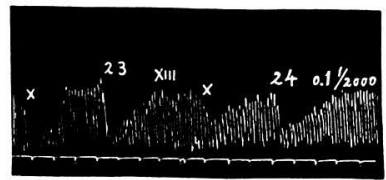

Fig. 1. f. XIII : Far weaker than 0.0001 mgrm. (Obs. 22); decidedly stronger than $0.00005 \mathrm{nigrm}$.

To sum up :

Specimen I : Stronger than $0.00005 \mathrm{mgrm}$; very far weaker than $0.0001 \mathrm{mgrm}$. per 0.5 c.c. It was assayed at $0.00015 \mathrm{mgrm}$. in 1 c.c.

Specimen III : Decidedly stronger than $0.00005 \mathrm{mgrm}$.; far weaker than $0.0001 \mathrm{mgrm}$. per 0.5 c.c. It was taken as $0.00015 \mathrm{mgrm}$. in 1 c.c.

Specimen V : A little stronger than $0.00005^{-}$mgrm.; far weaker than $0.0001 \mathrm{mgrm}$. per 0.5 c.c. It was taken as $0.00015 \mathrm{mgrm}$. in 1 c.c.

Specimen VIII : Stronger than $0.00005 \mathrm{mgrm}$.; almost as strong as or a little stronger than $0.00005 \mathrm{mgrm}$; weaker than $0.0001 \mathrm{mgrm}$. ( 2 assays) per 0.5 c.c. It was taken as $0.00015^{-}$mgrm. in 1 c.c.

Specimen X : Clearly stronger than $0.00005 \mathrm{mgrm}$; weaker than $0.0001 \mathrm{mgrm}$. per 0.5 c.c. It was assayed at $0.00015 \mathrm{mgrm}$. in 1 c.c.

Specimen XIII : Far stronger than $0.00005 \mathrm{mgrm}$; decidedly stronger than 0.00005 mgrm.; weaker than $0.0001 \mathrm{mgrm}$; far weaker than $0.0001 \mathrm{mgrm}$. per 0.5 c.c. It was taken as $0.00015 \mathrm{mgrm}$. in 1 c.c.

Blood flow, concentration of epinephrine and epinephrine output per min. per animal and per kilo of body weight are given in Table $I$.

\section{Example II.}

Dog 11. 우

22. XI. 1926. 10,0 kilos. $\mathrm{L}_{3}-\mathrm{D}_{9}$ dorsal spinal roots severed.

20. I. 1927. 11,8 kilos.

\begin{tabular}{|c|c|c|c|c|c|}
\hline Time & $\begin{array}{c}\text { Room } \\
\text { temper- } \\
\text { ature } \\
\left({ }^{\circ} \mathrm{C}\right)\end{array}$ & $\begin{array}{l}\text { Body } \\
\text { temper- } \\
\text { ature } \\
\left({ }^{\circ} \mathrm{C}\right)\end{array}$ & $\begin{array}{c}\text { Fre- } \\
\text { quency } \\
\text { of } \\
\text { respira- } \\
\text { tion } \\
\left(\begin{array}{c}\text { per } \\
\text { min. }\end{array}\right)\end{array}$ & $\begin{array}{c}\text { Rate of } \\
\text { heart } \\
\text { beat } \\
\left(\begin{array}{c}\text { per } \\
\text { min. }\end{array}\right)\end{array}$ & Experimental process and others \\
\hline $\begin{array}{l}\text { 9:30 a.m. } \\
10: 0\end{array}$ & 13.0 & & 18 & & $\begin{array}{l}\text { Dog was taken to operation room. } \\
\text { Lumbar route operation began. }\end{array}$ \\
\hline
\end{tabular}




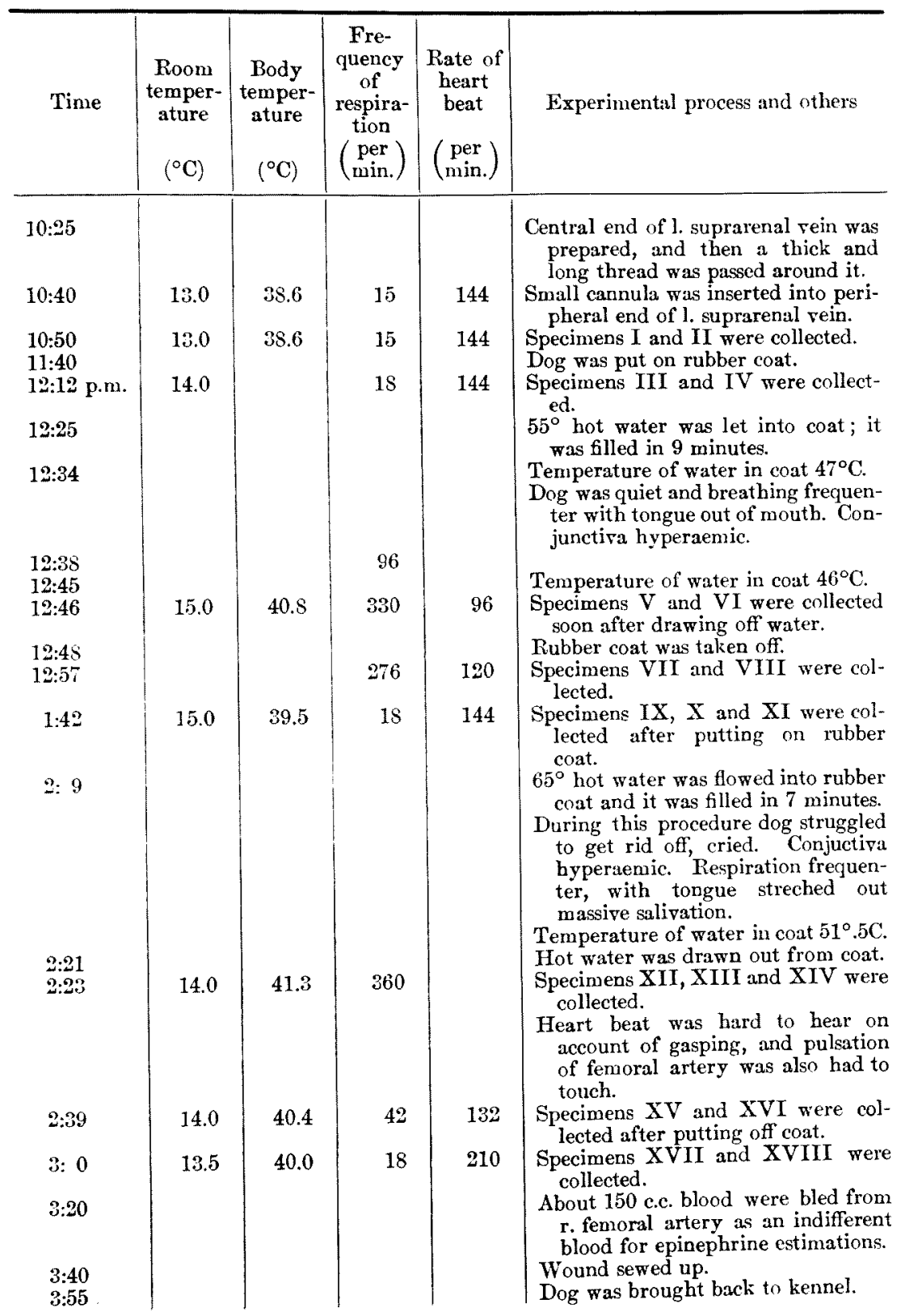


Intestine tracings* (Reduced to $\left.\frac{2}{5}\right):-$

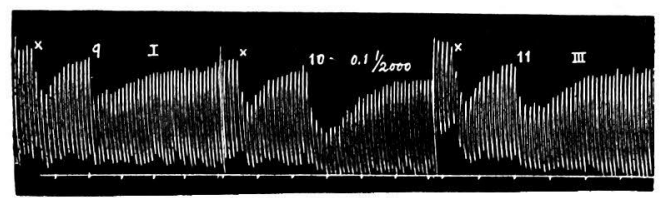

Fig. 2. a. I : Weaker than $0.00005 \mathrm{mgrm}$. III : Weaker than $0.00005 \mathrm{mgrm}$.

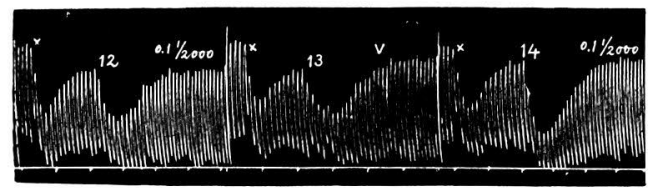

Fig. 2. b. III : Weaker than 0.00005 mgrm. (Obs. 11). V: $\Lambda$ little weaker than 0.00005 mgrm.

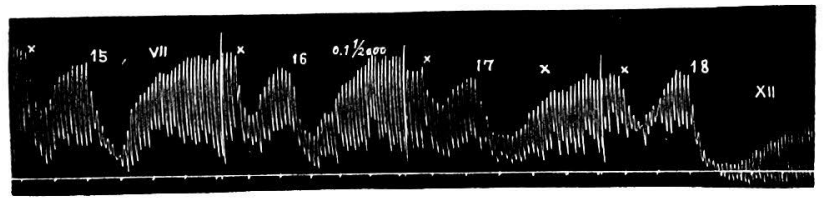

Fig. 2. c. VII : A very little weaker than $0.00005 \mathrm{mgrm}$. (Obs. 14 and 16). X : Very much stronger than $0.00005 \mathrm{mgrm}$. XII : Very much far stronger than X.

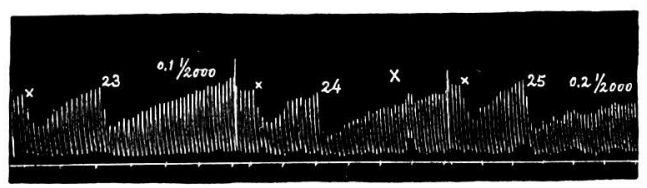

Fig. 2. d. An other new intestine segment was used. $\mathrm{X}$ : Very much stronger than $0.00005 \mathrm{mgrm}$; decidedly weaker than $0.0001 \mathrm{mgrm}$.

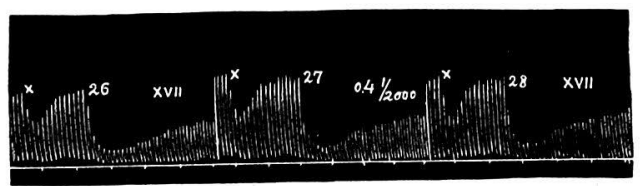

Fig. 2. e. XVII : Very fur stronger than $0.0001 \mathrm{mgrm}$. (Obs. 25); a little stronger than or almost as strong as $0.0002 \mathrm{mgrm}$.

* See the foot-note on p. 561. 


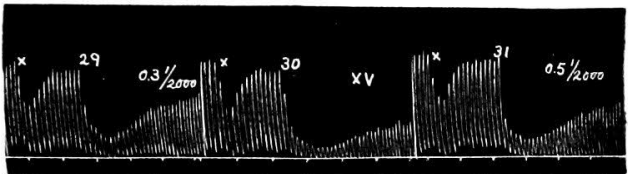

Fig. 2. f. XV : Very much stronger than $0.00015 \mathrm{mgrm}$; far stronger than 0.00025 mgrm. XVII : Much stronger than 0.00015 mgrm. (Obs. 28).

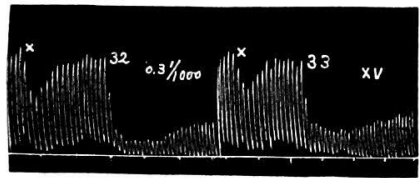

Fig. 2. g. XV: Weaker than $0.0003 \mathrm{mgrm}$.

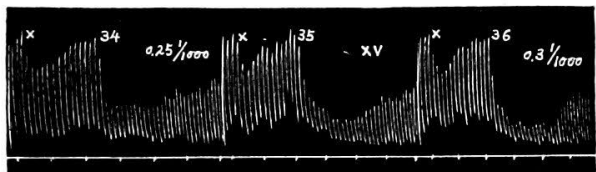

Fig. 2. h. XV : Stronger than $0.00025 \mathrm{mgrm}$. (Obs. 83 and 35 ); a little weaker than $0.0003 \mathrm{mgrm}$.

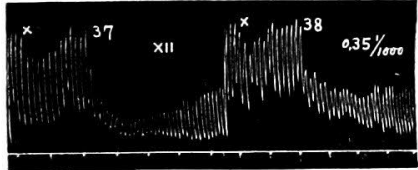

Fig. 2. i. XII : A little stronger than 0.0003 ingrm. (Obs. 36); stronger than 0.00035 mgrm.

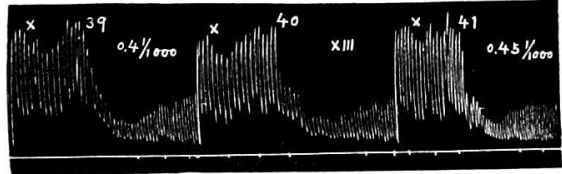

Fig. 2. j. XIII : Stronger than $0.0004 \mathrm{mgrm}$. and $0.00045 \mathrm{mgrm}$.

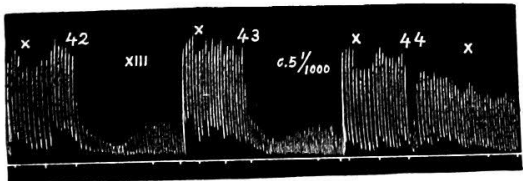

Fig. 2. k. XIII : Stronger than 0.00045 mgrm. (Obs. 41); a little weaker than 0.0005 mgrm. 
To sum up :

Specimen I: Weaker than $0.00005 \mathrm{mgrm}$. It was taken below than $0.0001 \mathrm{mgrm}$. in 1 c.c.

Specinen III : Weaker than $0.00005 \mathrm{mgrm}$. (2 assays). It was taken below than 0.0001 mgrm. in 1 c.c.

Specimen V: A little weaker than $0.00005 \mathrm{mgrm}$. It was assayed at weaker than $0.0001 \mathrm{mgrm}$. in 1 c.c.

Specimen VII : A very little weaker than $0.00005 \mathrm{mgrm}$. It was assayed at weaker than $0.0001 \mathrm{mgrm}$. in 1 c.c.

Specimen $\mathrm{X}$ : Very much stronger than 0.00005 mgrm. (2 assays); decidedly weaker than $0.0001 \mathrm{mgrm}$. It was taken as $0.00015 \mathrm{mgrm}$. in 1 c.c.

Specimen XIII : Stronger than $0.0004 \mathrm{mgrm}$. and $0.00045 \mathrm{mgrm}$. (2 assays); A little weaker than $0.0005 \mathrm{mgrm}$. It was assayed at $0.00095 \mathrm{mgrm}$. in $1 \mathrm{c.c}$.

Specimen XV : Very much stronger than $0.00015 \mathrm{mgrm}$; far stronger than 0.00025 mgrm. (2 assays); weaker than 0.0003 mgrm.; a little weaker than 0.0003 mgrm. It was assayed at $0.00055 \mathrm{mgrm}$. in 1 c.c.

Specimen XVII : Very far stronger than 0.0001 mgrm.; much stronger than 0.00015 mgrm.; a little stronger than or almost as strong as $0.0002 \mathrm{mgrm}$. It was assayed at 0.0004 mgrm. in 1 c.c.

Blood flow, concentration of epinephrine and epinephrine output per minute per animal and per kilo of body weight are given in Table II.

But when the temperature of water in the bath went higher, as $65^{\circ}$, the temperature of water in the coat accordingly ascended to $50^{\circ}$ or more and the rectal temperature fluctuated in $\operatorname{Dog} 9$ from $38.9^{\circ}$ to $40.5^{\circ}$, in Dog 10 from $39.0^{\circ}$ to $41.3^{\circ}$ and in Dog 11 from $39.5^{\circ}$ to $41.3^{\circ}$; namely in the last two dogs the body temperature became far higher than in the former series of heating experiments. In such cases the dog cried, struggled violently in order to get rid of the rubber coat, so that it was torn by scratching when the nails were not cut and the paws not bandaged. Faster breathing also occurred invariably, while the heart rate diminished a little. These findings on the pulse rate seem not to harmonize with those of various investigators, for example, those of $\mathrm{Ku}$ no on the urethanized rabbits. ${ }^{6}$ ) Abundant salivation occurred also.

The influence of heating upon the epinephrine output corresponded with the intensity of heating. When the heating was not excessive, it remained quite unaffected as in Dog 7 and the first set of experiments in Dog 11 , the blood flow rate and the epinephrine concentration during heating being found quite similar to those in the rest periods. In Dog 6, Dog 8 and $\operatorname{Dog} 9$ the epinephrine secretion was calculated as augmented because of the increase of either epinephrine concentration or blood flow, but the augmentation was by no means significant. Contrary to these, a remarkable acceleration in the rate of epinephrine discharge was seen in Dog 10 
and the last series of experiments on Dog 11 ; it resulted in a greater concentration of epinephrine. A bout seven times of the initial rate was noted. The subsequent samples, as specimens VII and VIII in Dog 10, which were collected 12 minutes after putting off the coat, and specimens XVXVI and XVIT-XVIII which were taken 16 and 40 minutes respectively after being freed from the coat, indicated that epinephrine continued still to be liberated at a somewhat faster rate, viz. a somewhat longer duration of after-action. The same is to be seen in Dog 6 (Specimens VII \& VIII) also.

In the instances where a notable acceleration in the epinephrine secretion was thus recorded, the anal temperature also increased notably, that is $41^{\circ}$ or more was measured, and the other reactions, as struggling, crying, etc., etc. were also excessive.

Summary:

The suprarenal vein bloods were collected from the non-anaesthetized, non-fastened dogs. The dorsal roots from IX. thoracic to III. lumbar cord were previously sectioned, and the lumbar route preparation was made. The epinephrine content was assayed by means of the rabbit intestine segment method.

1. Cooling of the dog was performed by means of a rubber cont, doubly layered, filled with cold water or by placing the animal's body in direct contact with an ice block. The anal temperature decreased $1.5^{\circ}$ or $1.7^{\circ}$ in some cases, the lowest temperature being estimated as $37^{\circ}$. No augmentation was noted in the rate of epinephrine discharge from the suprarenal gland.

2. Warming or heating of the auimal was carried out by means of the rubber coat. When the temperature in the coat was not so high, the epinephrine secretion rate remained unaltered, but when the temperature of the water in the coat was increased, the anal temperature ascended more, and when the latter exceeded about $41^{\circ} \mathrm{C}$ the reactions of the animal became excessive, and at the same time the velocity with which epinephrine was liberated increased notably, such as about seven times that during the rest spells. The after-action was fairly long. 